\title{
C01 | Assisted History Matching and Uncertainty Management
}

By Paul van den Hoek

Team Lead Quantitative Reservoir Management R\&D, Shell Innovation Research \& Development

\begin{abstract}
In the past years, we developed, improved and applied in the field a variety of different methodologies for assisted history matching (AHM) and improved uncertainty management, such as Experimental Design, Ensemble Kalman Filtering, Adjoint, streamlines. In most recent years, increasing emphasis has been on bringing these methodologies to our field operations, and also include 4D seismic into the history match process.

The current presentation will focus on how the different AHM methodologies were successfully applied in real field cases, including cases where production data were matched simultaneously with 4D seismic results.

A second topic of the presentation will be in the area "close the-big-loop" (fully geologically consistent) history matching where we are, amongst others, looking into developing methodologies for "business decision-relevant" modeling of reservoir uncertainties and multi-scales.
\end{abstract}

